Case Report

\title{
Large Subcapsular Splenic Hematoma with a Large Pancreatic Pseudocyst Was Successfully Treated with Splenic Arterial Embolization and Ultrasound-Guided Percutaneous Drainage of Pancreatic Pseudocyst
}

\author{
Song Zhang, Fei Liu, Heena Buch, Guifang Xu, and Lei Wang \\ Department of Gastroenterology, The Affiliated Drum Tower Hospital of Nanjing University Medical School, Nanjing, China \\ Correspondence should be addressed to Lei Wang; 867152094@qq.com
}

Received 6 May 2017; Revised 26 June 2017; Accepted 19 July 2017; Published 22 August 2017

Academic Editor: William B. Silverman

Copyright (C) 2017 Song Zhang et al. This is an open access article distributed under the Creative Commons Attribution License, which permits unrestricted use, distribution, and reproduction in any medium, provided the original work is properly cited.

\begin{abstract}
Subcapsular splenic hematoma is a rare complication of pancreatitis. The management for subcapsular splenic hematoma remains controversial. We herein report a case of a large subcapsular splenic hematoma with a large pancreatic pseudocyst, which was successfully treated with splenic arterial embolization and ultrasound- (US-) guided percutaneous drainage of pancreatic pseudocyst, for the first time. A 44-year-old male suffered from recurrent abdominal pain for more than two years. He had previous 3 episodes of pancreatitis. A subcapsular splenic hematoma $(16.0 \times 16.0 \times 7.6 \mathrm{~cm})$ with pancreatic pseudocyst $(13.5 \times 10.0 \times 8.0 \mathrm{~cm})$ was shown on abdominal computed tomography (CT). He underwent splenic arterial embolization to decrease the blood supply of the spleen and then ultrasound-guided percutaneous drainage of the large pancreatic pseudocyst. After 2 weeks, the repeated CT-Abdomen showed the disappearance of pancreatic pseudocyst and multiple areas of infarction on the spleen, while the splenic subcapsular hematoma had also significantly reduced. The patient was discharged after almost a month of his hospital admission with the drainage tube attached, and about 2 weeks later the drainage tube was removed upon CT scan confirmation of decrease in the volume of the subcapsular hematoma. Patient had no abdominal symptoms at the 1.5-year follow-up.
\end{abstract}

\section{Introduction}

A subcapsular splenic hematoma is a very rare hemorrhagic complication of acute or chronic pancreatitis. The prevalence of subcapsular hematoma of the spleen was estimated to be $0.4 \%$ in a recent study of 500 patients with chronic pancreatitis [1]. The management of this complication remains controversial. Thompson Jr. and Ashley [2] advocated early splenectomy to prevent rupture of the splenic hematoma and its associated morbidity. Rypens et al. [3] suggested that most of these complications could potentially regress and be managed conservatively. In addition, percutaneous drainage of the subcapsular splenic hematoma has been demonstrated in 4 case reports in the literature [4-7]. We herein present a case of a large subcapsular splenic hematoma with a large pancreatic pseudocyst successfully treated with splenic arterial embolization and US-guided percutaneous drainage of pancreatic pseudocyst, for the first time.

\section{Case Report}

A 44-year-old male was admitted to our hospital because of recurrent abdominal pain for more than two years that worsened over the past two weeks. He was a heavy drinker, with alcohol consumption of $250 \mathrm{~g} /$ day for 15 years. He had no history of trauma but previous 3 episodes of pancreatitis. $\mathrm{He}$ had the first episode of acute pancreatitis two years ago and received conservative treatment for it. The second episode of acute pancreatitis, about one and a half years ago, was accompanied by retroperitoneal cyst and splenomegaly along with dilation of the pancreatic duct. During the second episode, he underwent pancreatic duct dilation with a stent implanted into the pancreatic duct that was removed after 4 months. During the third episode of acute pancreatitis, about six months ago, a large pseudocyst was seen in the body and tail of the pancreas. Patient's symptoms were relieved after symptomatic treatment, but pseudocyst had not been 


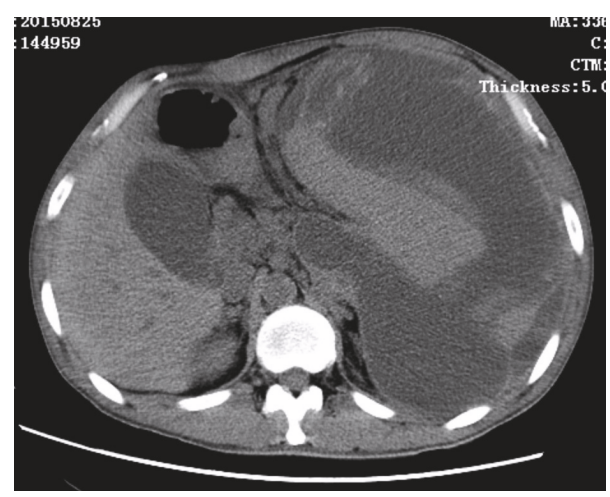

FIgURE 1: CT scan showing a large subcapsular splenic hematoma $(16.0 \times 16.0 \times 7.6 \mathrm{~cm})$ with a large pancreatic pseudocyst $(13.5 \times 10.0$ $\times 8.0 \mathrm{~cm})$.

treated. Nearly two weeks ago, the patient had recurrent upper abdominal pain, which was persistent and radiating to the left shoulder and back. He was admitted to local hospital. The routine blood test showed hemoglobin $102 \mathrm{~g} / \mathrm{L}$ and serum amylase $139 \mathrm{U} / \mathrm{L}$. Abdominal CT showed a large pseudocyst in the body and tail of the pancreas with signs of bleeding, a large subcapsular hematoma of the spleen, and cholecystitis with gallstones. Despite the conservative treatment consisting of pain control, bowel rest, intravenous fluids, and antibiotics, the pain was not relieved, so he visited our hospital.

After admission, his blood pressure, pulse rate, and body temperature were $117 / 69 \mathrm{mmHg}$, 58 beats per minute, and $36.5^{\circ} \mathrm{C}$, respectively. The abdominal examination revealed a palpable mass in the left upper quadrant with mild tenderness. The spleen was palpable under the left costal arch. Murphy's sign was negative. The laboratory findings showed that the liver function tests, renal function tests, and electrolytes were within normal limit. However, there was a leukocytosis $\left(12500 / \mathrm{mm}^{3}\right)$, increased C-reactive protein $(10.1 \mathrm{mg} / \mathrm{dL})$, and decreased hemoglobin $(9.1 \mathrm{~g} / \mathrm{dL})$ and hematocrit (26.9\%). The serum amylase was $117 \mathrm{U} / \mathrm{L}$, which were slightly above the normal range. The endoscopic ultrasonography revealed gastric vein varicosis in the fundus, pancreatic pseudocyst, cholecystitis with accumulation of biliary sludge, splenomegaly with subcapsular hematoma, and splenic vein varicosis. The CT-Abdomen revealed a huge low-density mass $(16.0 \times 16.0 \times 7.6 \mathrm{~cm})$ with some separations and loss of normal pancreatic morphology in the body and tail, splenomegaly with subcapsular hematoma $(13.5 \times 10.0 \times$ $8.0 \mathrm{~cm}$ ) that possibly pressurizes the left kidney, multiple cysts seen in the liver, gallstones, abdominal and pelvic effusion, bilateral pleural effusion with lungs atelectasis, splenic vein dilation, and expansion of portal vein branches (Figures 1-3).

During hospitalization, EUS-guided pseudocyst puncture and drainage were done, where small amount of dark brown liquid was collected. The following day CT-Abdomen showed no changes in the pancreatic pseudocyst and splenic subcapsular hematoma, while the NG tube was still in place (Figure 4). Splenic artery embolization (Figure 5) was done on the next day, with no significant changes on the follow-up CT-Abdomen result. About 4 days later, ultrasound-guided

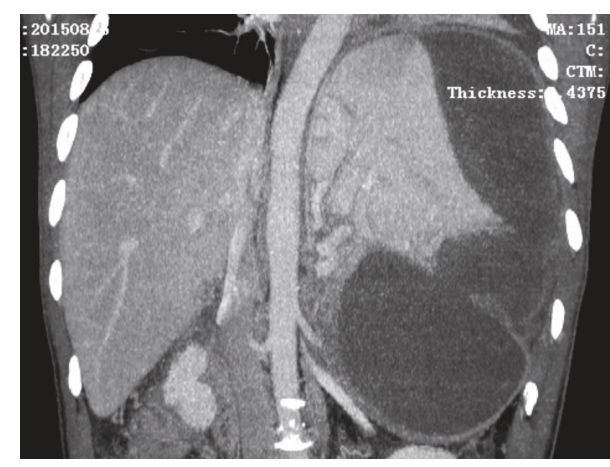

FIGURE 2: CT scan showing a large subcapsular splenic hematoma with a large pancreatic pseudocyst.

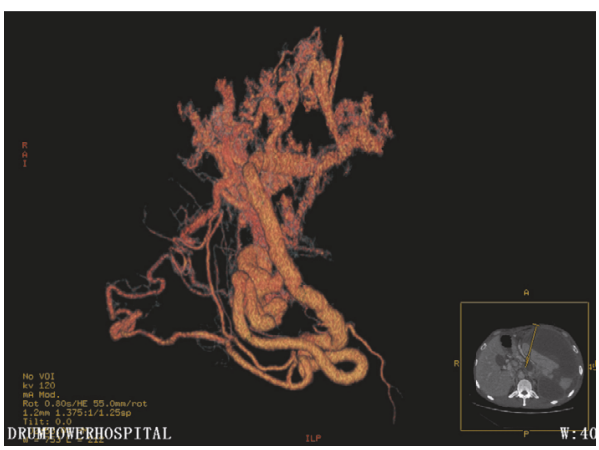

FIGURE 3: Vascular restructure with CT showing splenic vein dilation and expansion of portal vein branches.

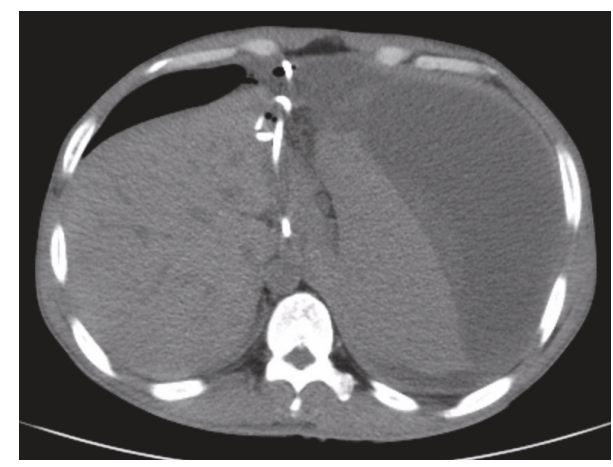

FIGURE 4: CT showing the drainage tube was in place, but splenic subcapsular hematoma had no change.

percutaneous puncture drainage of pancreatic pseudocyst was done (Figure 6). Postoperative fluid collection was about $5000 \mathrm{ml}$ of dark brown liquid with amylase $21577 \mathrm{U} / \mathrm{L}$, lipase $56704 \mathrm{U} / \mathrm{L}$, red blood cell count $0.45 \times 10^{12} / \mathrm{L}$, and hemoglobin $29 \mathrm{~g} / \mathrm{L}$. The follow-up CT-Abdomen (plain + contrast), about 2 weeks later, showed the disappearance of pancreatic pseudocyst and multiple areas of infarction on the spleen, while the splenic subcapsular hematoma had significantly reduced (Figure 7). Abdominal ultrasound was done to obtain detailed status of the subcapsular hematoma. During hospitalization, the patient was also supplemented by antibiotics, nutritional support, acid suppression, and 


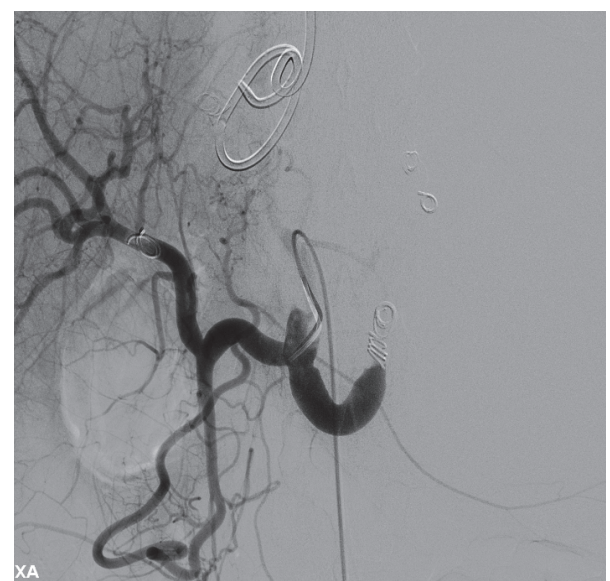

FIGURE 5: Showing coil embolization of splenic artery.

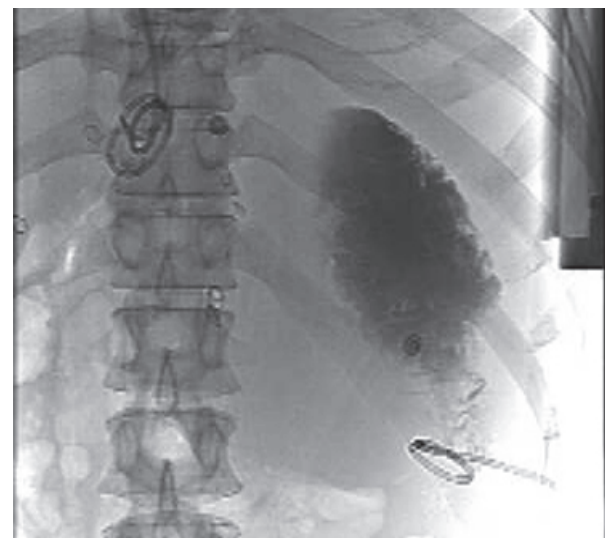

FIGURE 6: After US-guided percutaneous puncture drainage of pancreatic pseudocyst, X-ray showing drainage tube was in the right place.

inhibition of pancreatic secretion. The patient was discharged after almost a month of his hospital admission with the drainage tube attached, and about 2 weeks later the drainage tube was removed upon CT scan confirmation of decrease in the volume of the subcapsular hematoma (Figure 8). Patient had no abdominal symptoms at the 1.5 -year follow-up.

\section{Discussion}

In the present case, a large subcapsular splenic hematoma complicating large pancreatic pseudocyst gradually disappears after splenic arterial embolization and US-guided percutaneous drainage of a large pancreatic pseudocyst, and drainage of a subcapsular splenic hematoma and surgical procedures such as a splenectomy and distal pancreatectomy were avoided.

Splenic hematoma is a rare complication of acute or chronic pancreatitis when compared with traumatic origin of subcapsular hematoma. The tail of the pancreas is in close proximity to the splenic hilum, whereas the peritoneum encases the anterior pancreatic surface along with the splenic vessels $[8,9]$. This anatomy provides a potential

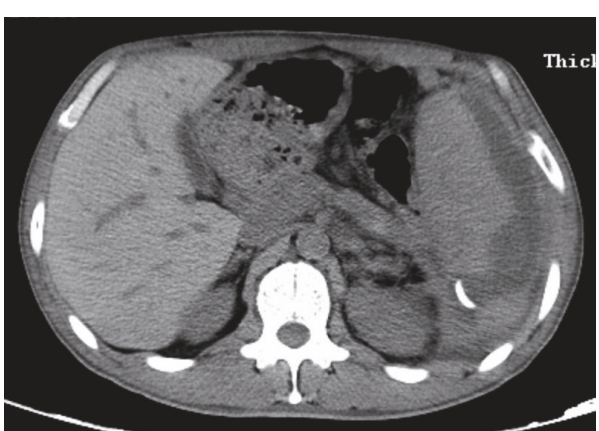

Figure 7: About 2 weeks after splenic artery embolization and ultrasound-guided percutaneous drainage of pancreatic pseudocyst, CT showing the disappearance of pancreatic pseudocyst, multiple areas of infarction on the spleen, and the significant reduction of splenic subcapsular hematoma.

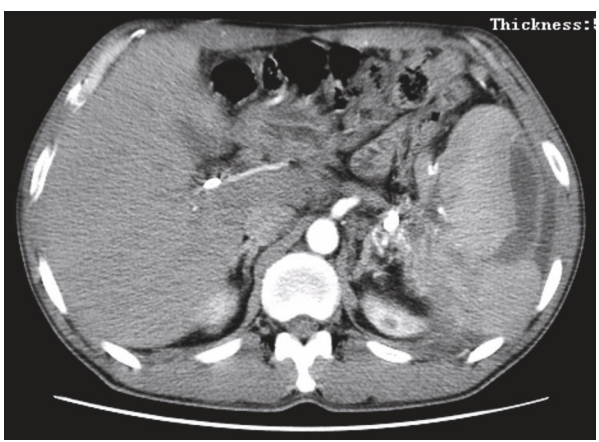

FIGURE 8: CT scan showing the volume of splenic subcapsular hematoma significantly reduced compared to all previous images.

for splenic involvement in pancreatitis. There are several possible theories which might explain the mechanisms that how pancreatitis leads to subcapsular splenic hematoma: (1) erosion of splenic parenchyma after pancreatic enzyme leakage, resulting in either a splenic hematoma or intrasplenic bleeding, (2) disruption of splenic hilar vessels leading to subcapsular splenic hematoma, and (3) expansion of intrasplenic pseudocysts resulting in splenic rupture and bleeding [8]. In our case report, patient has a history of three episodes of pancreatitis. During the third episode, the CT scan showed pseudocysts with variable sizes at the body and tail of the pancreas, which were compressing the splenic vein and parenchyma. Therefore, according to the results of CT scan and enhancement imaging, we assume that direct erosion into the splenic parenchyma or disruption of the splenic vein might have led to a subcapsular splenic hematoma in this case.

Because splenic involvement in patients with pancreatitis is uncommon, the diagnosis of subcapsular hematoma of the spleen needs the alertness of physicians and imaging studies. Patients with pancreatitis exhibiting a mass in the left upper quadrant, pain radiating to the left shoulder, elevation of the left diaphragm, and a moderate fall in hematocrit should be suspected to have splenic complications. Imaging studies that are used to monitor the pancreatitis, such as 
CT and MRI, are regarded as reliable diagnostic tools and should be performed early in questionable patients. A splenic hematoma can be distinguished from simple fluid collection based on density (Hounsfield units $>30$ ). Angiography is not essential for the diagnosis but would be indicated if splenic artery pseudoaneurysm, splenic vein thrombosis, or active bleeding was suspected $[2,6]$.

The management of subcapsular splenic hematoma in pancreatitis remains controversial, maybe due to its rareness. Some reports have advocated aggressive management with early splenectomy to avoid splenic rupture $[1,2,10]$. Thompson Jr. and Ashley [2] described 3 cases with acute pancreatitis accompanied by large subcapsular hematoma of the spleen. Two patients recovered after splenectomy, but 1 patient died due to continuing blood loss. They suggested that the treatment of subcapsular splenic hematoma should be a splenectomy in the vast majority of patients to prevent continuing blood loss and potential rupture. However, some authors advocate a conservative approach, which is based on the experience with traumatic lesions that can resolve spontaneously. Patel et al. [8] reported a case of subcapsular splenic hematoma managed conservatively with a favorable outcome, in which the CT scan showed the marked resolution of the hematoma after 4 months. This approach was considered as a safe management in hemodynamically stable patients. As a part of conservative approaches, percutaneous drainage of the hematoma, which showed good prognosis, may be increasingly used in recent years. Four cases of a subcapsular splenic hematoma have been reported which were successfully treated by percutaneous drainage of the hematoma [4-7]. Prompt relief of symptoms, a short recovery time, avoidance of rupture, and spleen preservation are the benefits of percutaneous drainage. In addition, Purushothaman and Borowski [11] also demonstrated the satisfying effect of splenic artery embolization for subcapsular splenic hematoma. However, it was just one particular case, which was reported.

In this special case, the patient suffered from a large subcapsular splenic hematoma with a large pancreatic pseudocyst. After repeatedly discussing about patient history and imaging studies in the multidisciplinary team (MDT), we considered that the only drainage of large pancreatic pseudocyst or spleen subcapsular hematoma will destroy the balance of the existing abdominal pressure, which may worsen subcapsular splenic hematoma, and the rupture of splenic hematoma and other unpredictable life-threatening consequences might even occur. Therefore, we made splenic artery embolization to decrease the blood supply of the spleen. Following, we made the US-guided percutaneous drainage of the large pancreatic pseudocysts. The postoperative abdomen CT scan demonstrated the significant resolution of pancreatic pseudocyst and subcapsular splenic hematoma, with relief of symptoms. There are several possibilities which might explain that subcapsular splenic hematoma gradually disappeared after splenic artery embolization and percutaneous drainage of the large pancreatic pseudocysts: (1) splenic artery embolism reduces the blood supply of spleen, and spleen subcapsular bleeding stopped; (2) after adequate percutaneous drainage of the large pancreatic pseudocyst, the pressure of splenic hilum decreased and the return of splenic vein increased which accelerated the absorption of hematoma; (3) there was the common channel between subcapsular splenic hematoma and pancreatic pseudocyst, so subcapsular splenic hematoma was also being drained while draining the pancreatic pseudocyst.

In conclusion, the definitive management of a subcapsular splenic hematoma complicating pancreatic pseudocyst is not yet established. Surgical intervention may be one of the therapeutic options for hemodynamically unstable patients, but with high mortality rate. Imaging-guided percutaneous drainage and splenic artery embolization appeared to be another feasible option for subcapsular splenic hematomas to prevent splenic rupture and obviate splenectomy. For the case of subcapsular splenic hematoma with a large pancreatic pseudocyst, the splenic artery embolization and US-guided percutaneous drainage of large pancreatic pseudocyst may be a safe and effective management.

\section{Conflicts of Interest}

The authors declare that they have no conflicts of interest.

\section{Authors' Contributions}

Song Zhang and Fei Liu contributed equally to this article.

\section{References}

[1] D. Malka, P. Hammel, P. Lévy et al., "Splenic complications in chronic pancreatitis: Prevalence and risk factors in a medicalsurgical series of 500 patients," Gastroenterology, vol. 85, pp. $1645-1649,1998$.

[2] J. E. Thompson Jr. and S. W. Ashley, "Subcapsular hematoma of the spleen associated with acute pancreatitis," Surgery, vol. 121, no. 2, pp. 231-233, 1997.

[3] F. Rypens, J. Devière, M. Zalcman et al., "Splenic parenchymal complications of pancreatitis: CT findings and natural history," Journal of Computer Assisted Tomography, vol. 21, no. 1, pp. 8993, 1997.

[4] C. J. Vyborny, T. N. Merrill, J. Reda, R. E. Geurkink, and S. J. Smith, "Subacute subcapsular hematoma of the spleen complicating pancreatitis: Successful percutaneous drainage," Radiology, vol. 169, no. 1, pp. 161-162, 1988.

[5] T. L. T. Siu, "Percutaneous drainage of spontaneous subcapsular haematoma of the spleen complicating chronic pancreatitis," Surgeon, vol. 2, no. 1, pp. 52-55, 2004.

[6] C.-W. Tseng, C.-C. Chen, J.-H. Chiang, F.-Y. Chang, H.-C. Lin, and S.-D. Lee, "Percutaneous drainage of large subcapsular hematoma of the spleen complicating acute pancreatitis," Journal of the Chinese Medical Association, vol. 71, no. 2, pp. 92-95, 2008.

[7] Y.-I. Kim, S.-Y. Park, J.-H. Lee et al., "A case of pancreatic pseudocyst with a large subcapsular splenic hematoma treated successfully by ultrasonography-guided percutaneous drainage," The Korean Journal of Gastroenterology, vol. 57, no. 4, pp. 258-261, 2011.

[8] V. G. Patel, O. M. Eltayeb, M. Zakaria, J. K. Fortson, and W. L. Weaver, "Spontaneous subcapsular splenic hematoma: a rare 
complication of pancreatitis," The American Surgeon, vol. 71, no. 12, pp. 1066-1069, 2005.

[9] V. Gandhi, S. Philip, A. Maydeo et al., "Ruptured subcapsular giant haematoma of the spleen-a rare complication of acute pancreatitis," Trop Gastroenterol, vol. 31, 123, p. 124, 2010.

[10] A. Agnifili, F. Gianfelice, P. Gola et al., "Subcapsular splenic hematoma complicating chronic relapsing pancreatitis. Case report," Acta Chirurgica - European Journal of Surgery, vol. 157, no. 1, pp. 63-65, 1991.

[11] K. Purushothaman and D. W. Borowski, "Unusual presentation of spontaneous splenic haematoma due to severe pancreatitis: a cautionary tale," BMJ Case Reports, vol. 2012, 2012. 


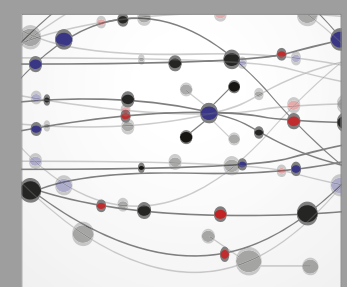

The Scientific World Journal
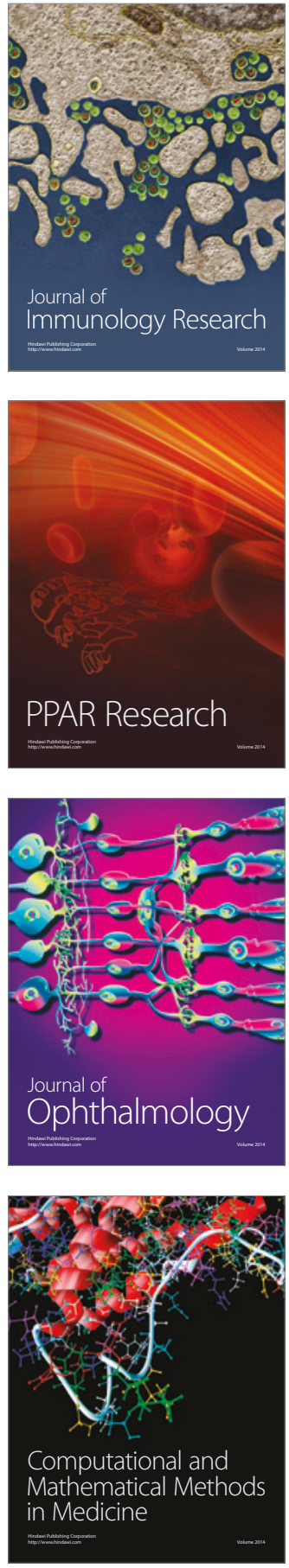

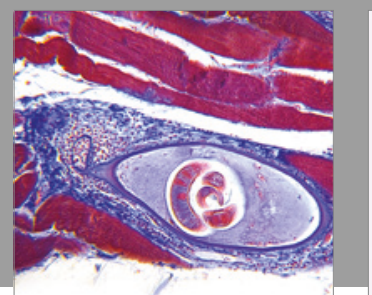

Gastroenterology Research and Practice
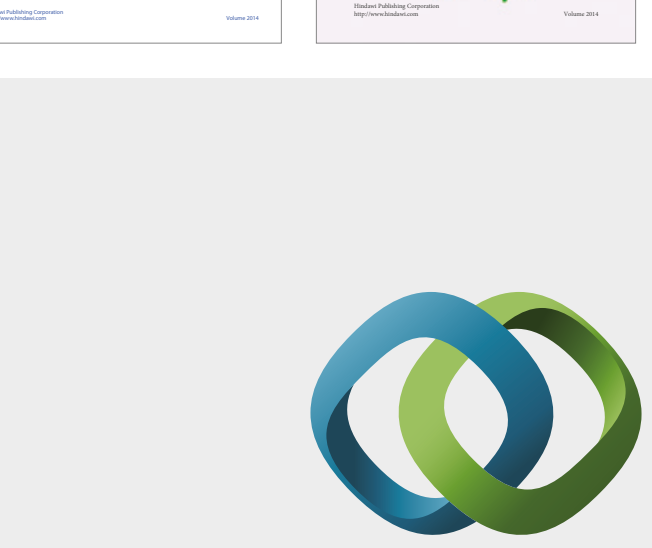

\section{Hindawi}

Submit your manuscripts at

https://www.hindawi.com
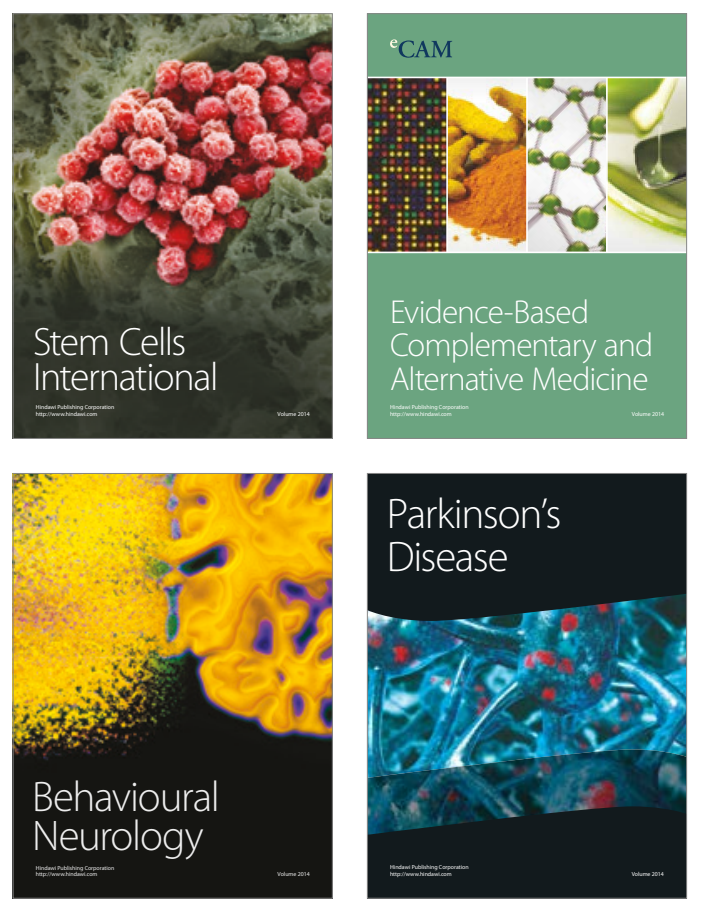
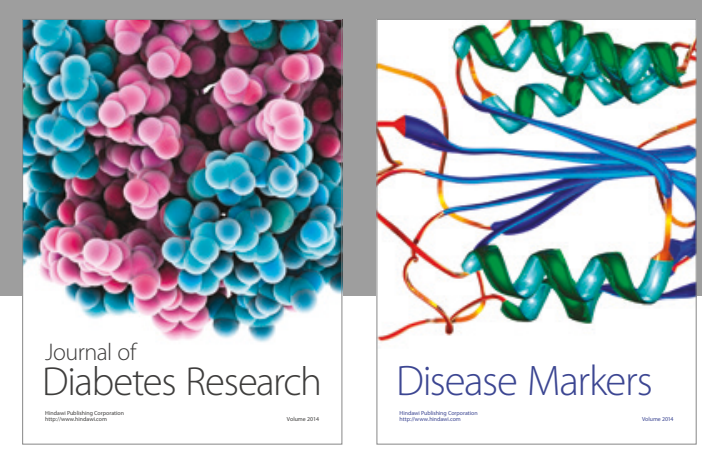

Disease Markers
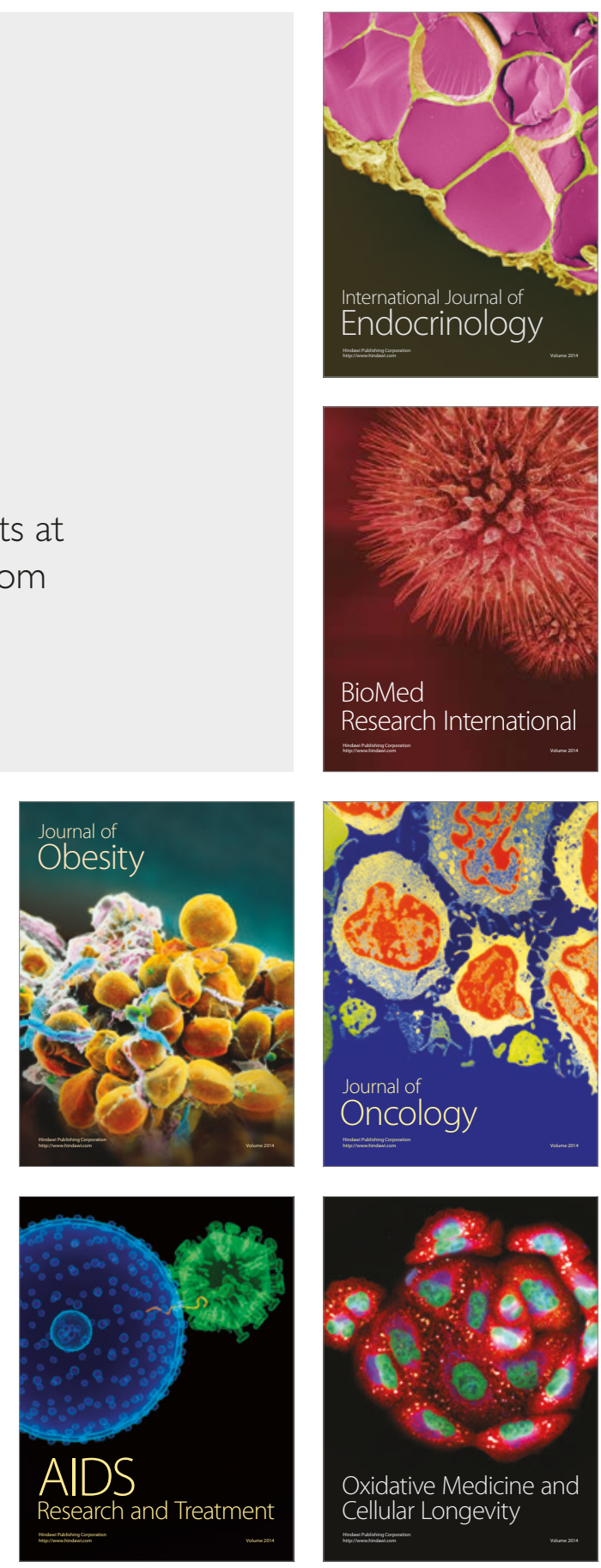